\section{Photodynamic
therapy: current role in
the treatment of
chorioretinal
conditions}

Department of

Ophthalmology, Addenbrooke's Hospital,

Cambridge University Hospitals NHS Foundation Trust, Cambridge, UK

Correspondence: DK Newman, Department of Ophthalmology (Box 41), Addenbrooke's Hospital, Cambridge University Hospitals NHS Foundation Trust, Hills Road, Cambridge, CB2 0QQ, UK Tel: +44 (0)12 2324 5151; Fax: +44 (0)12 23217968 E-mail: douglas.newman@ addenbrookes.nhs.uk

Received: 27 September 2015 Accepted: 30 September 2015 Published online:

8 January 2016

This paper was presented at the 45th Cambridge

Ophthalmological

Symposium,

September 2015.

\begin{abstract}
Verteporfin photodynamic therapy (vPDT) is a selective vaso-occlusive treatment that targets choroidal vascular abnormalities. It was initially developed to treat neovascular age-related macular degeneration using the 'standard' vPDT protocol (verteporfin $6 \mathrm{mg} /$ $\mathrm{m}^{2}$, vPDT laser fluence $50 \mathrm{~J} / \mathrm{cm}^{2}$ ). vPDT therapy has subsequently evolved as an important treatment modality for a range of other chorioretinal conditions including choroidal haemangioma, central serous chorioretinopathy, polypoidal choroidal vasculopathy, and peripapillary choroidal neovascularisation. Various 'safety-enhanced' vPDT protocols have been devised to optimise treatment outcomes, typically using reduced dose verteporfin (verteporfin $3 \mathrm{mg} / \mathrm{m}^{2}$ ) or reduced fluence vPDT (vPDT laser fluence $25 \mathrm{~J} / \mathrm{cm}^{2}$ ). This paper reviews the current role of vPDT therapy in the treatment of chorioretinal conditions.
\end{abstract}

Eye (2016) 30, 202-210; doi:10.1038/eye.2015.251; published online 8 January 2016

\section{Introduction}

Photodynamic therapy (PDT) is a therapeutic technique involving the use of a photosensitising agent that produces selective tissue damage when activated by light of a specific wavelength.

PDT was initially developed to treat cancer by selective destruction of tumour cells and/or tumour vasculature. ${ }^{1,2}$ The development of improved photosensitising agents in the 1980s enabled effective PDT therapy for a variety of tumours with minimal adverse effects (notably reduced skin phototoxicity). PDT is currently approved in oncology as a topical therapy for non-melanoma skin cancer (aminolevulinic acid
DK Newman

or methyl aminolevulinate) and as a systemic therapy for non-small cell lung cancer and oesophageal cancer (porfimer sodium) and advanced head and neck squamous cell cancer (temoporfin). ${ }^{3}$

Ophthalmic PDT was developed in the 1990s as a treatment for subfoveal choroidal

neovascularisation (CNV) in age-related macular degeneration (AMD). Encouraging results in animal models of CNV led to human clinical trials for three photosensitising agents: benzoporphyrin derivative (verteporfin), tin ethyl etiopurpurin (purlytin) and lutetium texaphyrin (Lu-tex). ${ }^{4}$ Verteporfin emerged as the optimal agent because of its absorption spectrum, lipophilic characteristics and short serum half-life (minimising the duration of skin photosensitivity). ${ }^{5,6}$ This review will cover the current role of verteporfin PDT (vPDT) therapy in the treatment of chorioretinal conditions.

\section{Mechanism of action}

vPDT therapy produces selective vascular occlusion by targeting vascular endothelial cells. Verteporfin was developed to preferentially accumulate in abnormal neovascular endothelial cells through their increased expression of low-density lipoprotein (LDL) receptors compared to normal choroidal and retinal vessels. ${ }^{7}$ Verteporfin is administered as a liposomal formulation, which may further enhance its relative selectivity for abnormal neovascularisation.

Verteporfin produces a photochemical reaction when activated by light corresponding to its absorption spectrum. Verteporfin has a broad absorption spectrum, but only the far-red peak at $689 \mathrm{~nm}$ is utilised in clinical practice. The use of far-red wavelength light allows good penetration through melanin, blood and fibrotic 
tissue enabling effective treatment of pigmented or haemorrhagic lesions located within the choroid. ${ }^{8}$ The vPDT laser delivery system provides localised light irradiation of the target lesion.

The photochemical reaction is complex, depending on the local tissue microenvironment. Following light irradiation, verteporfin is excited from its ground state to a higher energy state. This activated verteporfin molecule interacts with oxygen and/or biological substrates, leading to the creation of cytotoxic singlet oxygen and free radicals. These highly reactive species have a very short diffusion pathway enabling localised cell damage and death. vPDT therapy produces vascular endothelial cell damage that leads to platelet aggregation, activation of the clotting cascade and microvascular occlusion. ${ }^{8}$

The efficacy and selectivity of vPDT therapy for choroidal vascular lesions is dependent on several factors: verteporfin dosage, duration of verteporfin infusion and light dose delivered. ${ }^{8,9}$ Phase I/II clinical studies in neovascular AMD demonstrated that the minimum effective light dose is greater than $25 \mathrm{~J} / \mathrm{cm}^{2}$ and the maximum tolerated light dose (defined by retinal vessel closure) is less than $150 \mathrm{~J} / \mathrm{cm}^{2}{ }^{5}$ Tissue selectivity can also be influenced by the time interval between verteporfin administration and light irradiation. ${ }^{9}$ The timing of light irradiation should be selected so that verteporfin is at its maximal concentration in the target tissue compared to surrounding normal tissue.

\section{vPDT treatment protocols}

vPDT is a two-step procedure that involves intravenous infusion of verteporfin followed by application of 689-nm red laser light to the target lesion. The following 'standard' vPDT protocol was developed for the TAP and VIP studies to treat neovascular AMD with subfoveal CNV. ${ }^{10,11}$

Verteporfin is administered by intravenous infusion over $10 \mathrm{~min}$ at a dose of $6 \mathrm{mg} / \mathrm{m}^{2}$ body surface area (BSA). A short wait of $5 \mathrm{~min}$ is allowed for selective localisation of verteporfin in the target lesion and clearance of drug from surrounding normal tissue. Verteporfin is then activated by exposure to low-energy (non-thermal) laser light. vPDT lasers deliver red light at a wavelength of $689 \mathrm{~nm}$. The following laser parameters are used for the standard vPDT protocol: fluence $50 \mathrm{~J} / \mathrm{cm}^{2}$, irradiance $600 \mathrm{~mW} / \mathrm{cm}^{2}$, duration $83 \mathrm{~s}$.

The vPDT laser delivery system produces a circular spot of light. A slit-lamp biomicroscope is used to visualise the fundus with a standard laser contact lens. The diameter of the laser spot can be varied according to the size of the target lesion (determined by angiography). The laser spot is adjusted to exceed the greatest linear dimension (GLD) of the target lesion (typically by a margin of $500 \mu \mathrm{m})$. The maximum laser spot size generated by most vPDT laser delivery systems is around $7000 \mu \mathrm{m}$.

vPDT therapy has a good safety profile. No serious safety concerns were identified during the TAP and VIP studies. ${ }^{10,11}$ The most frequent adverse events are visual disturbances (10-15\%), photosensitivity reactions (3\%), and back pain during the verteporfin infusion $(2 \%)$.

Extravasation of verteporfin is the most serious complication as it can lead to severe skin necrosis if not properly treated. Following vPDT therapy, all patients should be advised to avoid exposure to sunlight for $48 \mathrm{~h}$ because of skin photosensitivity.

Various protocols have been developed in order to enhance the efficacy and safety profile of vPDT therapy. These vPDT protocol variations are based on the doseresponse-dependent nature of the photochemical reaction. The most widely used 'safety-enhanced' vPDT protocols utilise reduced dose verteporfin (typically verteporfin $3 \mathrm{mg} / \mathrm{m}^{2} \mathrm{BSA}$ ) or reduced fluence vPDT (typically vPDT laser fluence $25 \mathrm{~J} / \mathrm{cm}^{2}$ ). There is limited evidence to determine the optimum vPDT therapy parameters for different chorioretinal conditions. ${ }^{12-15}$

\section{Neovascular age-related macular degeneration}

vPDT therapy was developed to treat neovascular AMD with subfoveal CNV. Phase I/II clinical studies demonstrated that a single treatment achieved effective CNV closure, but reperfusion typically occurred after 6-12 weeks. ${ }^{5}$ Multiple applications of vPDT enabled longer term CNV control without significantly increased adverse effects. ${ }^{6}$ This strategy was adopted in randomised clinical trials to investigate the efficacy of vPDT therapy for neovascular AMD.

The TAP study (published 2001) demonstrated that vPDT therapy was a moderately effective treatment for predominantly classic CNV lesions. ${ }^{10}$ It significantly reduced the risk of moderate and severe visual loss in this group of patients for at least 2 years. The VIP study (published 2001) demonstrated that vPDT therapy provided marginal benefit in the treatment of occult CNV lesions (with recent disease progression). ${ }^{11}$ Subgroup analysis suggested a greater benefit for relatively small lesions or larger lesions with relatively poor visual acuity. The VIM study (published 2005) demonstrated a marginal benefit for vPDT therapy in the treatment of relatively small minimally classic CNV lesions. ${ }^{12}$

The standard vPDT therapy protocol was established by the TAP and VIP studies. Following baseline vPDT treatment, patients are reviewed every 3 months with repeat vPDT treatment if the CNV lesion reactivates as judged by leakage on fluorescein angiography. Repeat vPDT treatments are applied using a laser spot size 
corresponding to the recurrent area of leakage (plus a margin of $500 \mu \mathrm{m}$ ). This follow-up process is continued indefinitely until the CNV lesion has stabilised (ie inactive CNV lesion with minimal fluorescein leakage and stable visual acuity). Most patients experience some visual deterioration during their course of vPDT therapy, which usually occurs during the first 12 months.

The treatment of neovascular AMD underwent a paradigm shift following the MARINA and ANCHOR studies (published 2006). ${ }^{16-18}$ Anti-VEGF therapy with ranibizumab was demonstrated to be an effective treatment for all types of subfoveal CNV lesion. This treatment modality stabilised vision in most patients and, furthermore, improved vision in $\sim 30 \%$ of patients. Anti-VEGF therapy was rapidly adopted as the standard of care for neovascular AMD since it was much more effective than vPDT therapy.

There has been some interest in combination therapy using ranibizumab and vPDT to treat neovascular AMD. Combining treatment modalities with differing mechanisms of action may have synergistic effects, thereby leading to improved outcomes. It was proposed that vPDT might reduce the frequency of ranibizumab injections, which represents a significant treatment burden with anti-VEGF monotherapy. Unfortunately, combination therapy was found to provide no significant benefit in the MONT BLANC and DENALI studies (published 2012). ${ }^{19,20}$ The RADICAL study also found no significant benefit from triple therapy using vPDT, ranibizumab, and dexamethasone (ClinicalTrials.gov Identifier NCT00492284, September 2015).

Currently, there is a very limited role for vPDT therapy in the treatment of neovascular AMD. vPDT therapy can be used as rescue therapy in patients not responding to anti-VEGF monotherapy. ${ }^{21}$ It is, however, also important to consider alternative diagnoses in anti-VEGF non-responders (in particular chronic central serous chorioretinopathy or polypoidal choroidal vasculopathy), which might respond to different treatment modalities such as vPDT. vPDT therapy can occasionally be useful to treat patients unable to tolerate the intravitreal injection procedure required for anti-VEGF therapy. When treating neovascular AMD, reduced fluence vPDT $\left(25 \mathrm{~J} / \mathrm{cm}^{2}\right)$ may possibly reduce the risk of treatment-related visual loss compared with standard fluence vPDT $\left(50 \mathrm{~J} / \mathrm{cm}^{2}\right) .{ }^{12}$

\section{Non-AMD choroidal neovascularisation}

vPDT therapy has also been investigated for the treatment of non-AMD causes of subfoveal CNV such as pathological myopia, angioid streaks, presumed ocular histoplasmosis syndrome, and idiopathic CNV. ${ }^{22}$

The VIP study investigated the safety and efficacy of vPDT therapy for myopic CNV. It reported a significantly better visual outcome at 12 months, ${ }^{23}$ but no significant visual benefit at 24 months. ${ }^{24}$ The failure to demonstrate long-term efficacy is probably due to vPDT-related chorioretinal atrophy in a condition that tends to regress spontaneously. A longer term study did suggest some benefit in patients with better initial visual acuity and larger lesions in younger patients. ${ }^{25}$ More recent clinical trials have, however, demonstrated that anti-VEGF therapy achieves much better visual outcomes for myopic CNV than vPDT therapy. ${ }^{26-28}$ Anti-VEGF therapy is currently the standard of care for treating myopic CNV. ${ }^{29}$

vPDT therapy has been used as a treatment for other causes of CNV with variable outcomes. Guidelines were published at an early stage based on the clinical trial experience of vPDT therapy for neovascular AMD and myopic CNV. ${ }^{30}$ The evidence base for vPDT therapy in other causes of CNV has necessarily been developed from published case reports and small case series. It is not feasible to conduct clinical trials owing to the infrequent occurrence of these conditions. In more recent years, antiVEGF therapy has largely replaced vPDT therapy as the preferred treatment modality for these other causes of $\mathrm{CNV}$ as it achieves much better visual outcomes (similar to the situation with myopic $\mathrm{CNV}) .{ }^{31,32}$

\section{Choroidal haemangioma}

Choroidal haemangiomas require treatment when vision is affected by macular oedema or exudative retinal detachment. Asymptomatic lesions can be safely observed since there is a low risk of progression with visual deterioration. Various techniques have been used to treat choroidal haemangiomas in the past including laser photocoagulation, transpupillary thermotherapy, and radiation therapy (external beam, plaque brachytherapy, or proton beam). vPDT therapy is now considered to be the treatment of choice for this condition.

In many respects, choroidal haemangioma can be regarded as a prototypic disease for vPDT therapy owing to the characteristics of its vascular structure. Verteporfin is sequestered in the abnormal large calibre vessels with limited accumulation in normal choriocapillaris. vPDT therapy therefore enables selective targeting of the tumour vessels with minimal damage to the overlying neurosensory retina. Multiple case series have been reported demonstrating that vPDT therapy achieves high rates of tumour control with minimal complications. ${ }^{33-38}$

Choroidal haemangiomas can be effectively treated using the standard vPDT protocol-verteporfin dosage $6 \mathrm{mg} / \mathrm{m}^{2}$ BSA and vPDT laser fluence $50 \mathrm{~J} / \mathrm{cm}^{2}$ $\left(600 \mathrm{~mW} / \mathrm{cm}^{2}\right.$ for $\left.83 \mathrm{~s}\right){ }^{36,37}$ Alternative vPDT protocols have been reported, generally involving a higher vPDT laser fluence of $100 \mathrm{~J} / \mathrm{cm}^{2}$ (600 mW/ $\mathrm{cm}^{2}$ for $\left.166 \mathrm{~s}\right){ }^{33-35,38}$ The maximum laser spot size generated by most vPDT 
laser systems is around $7000 \mu \mathrm{m}$. Consequently, treatment of larger choroidal haemangiomas may require multiple, partially overlapping laser spots (administered during the same treatment session).

The majority of patients with choroidal haemangioma require only one vPDT treatment session in order to achieve long term tumour control (Figure 1 a.1-a.5). The end point of treatment is generally considered to be elimination of exudation rather than complete tumour regression. There is usually some reduction in tumour thickness following vPDT therapy. Complete tumour regression generally requires more aggressive vPDT therapy with a potential risk of adverse effects such as choroidal atrophy and neurosensory retinal degeneration. ${ }^{33}$

Radiation therapy remains a useful treatment modality for larger choroidal haemangiomas with extensive exudative retinal detachment that are not amenable to vPDT therapy. ${ }^{39}$ There is, however, a risk of radiationinduced complications (cataract, radiation retinopathy and optic neuropathy). Diffuse choroidal haemangiomas associated with Sturge-Weber syndrome can be treated by vPDT therapy, ${ }^{40}$ though radiation therapy may be required for more severe cases.

\section{Central serous chorioretinopathy}

Central serous chorioretinopathy (CSC) is an idiopathic chorioretinal condition. The aetiology is incompletely understood but a principal feature is localised choroidal vascular hyperpermeability. Breakdown of the retinal pigment epithelial (RPE) barrier leads to leakage of fluid into the subretinal space with development of a macular neurosensory detachment. CSC can be broadly classified
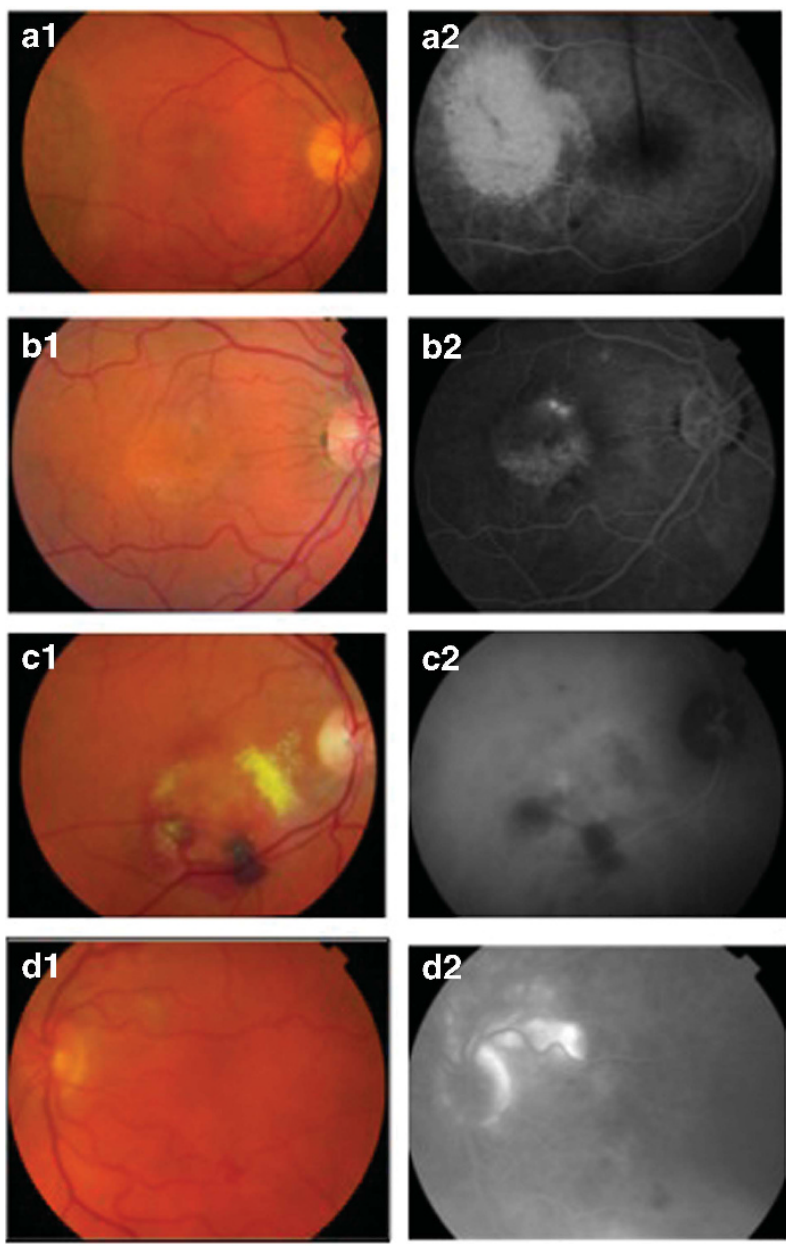
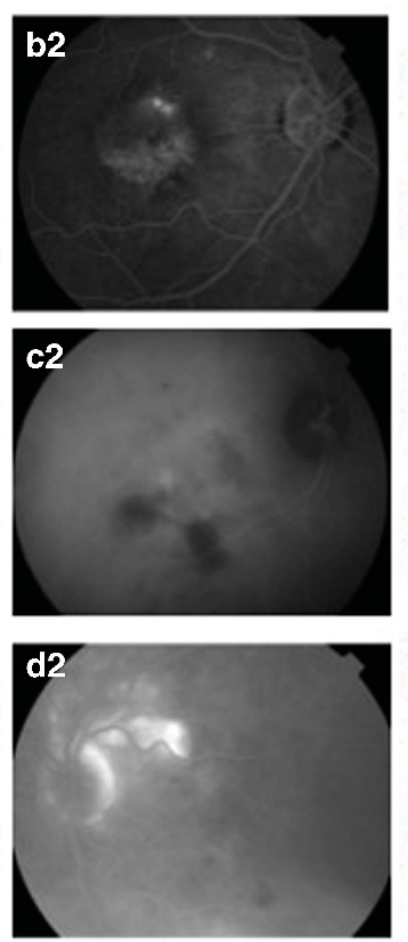
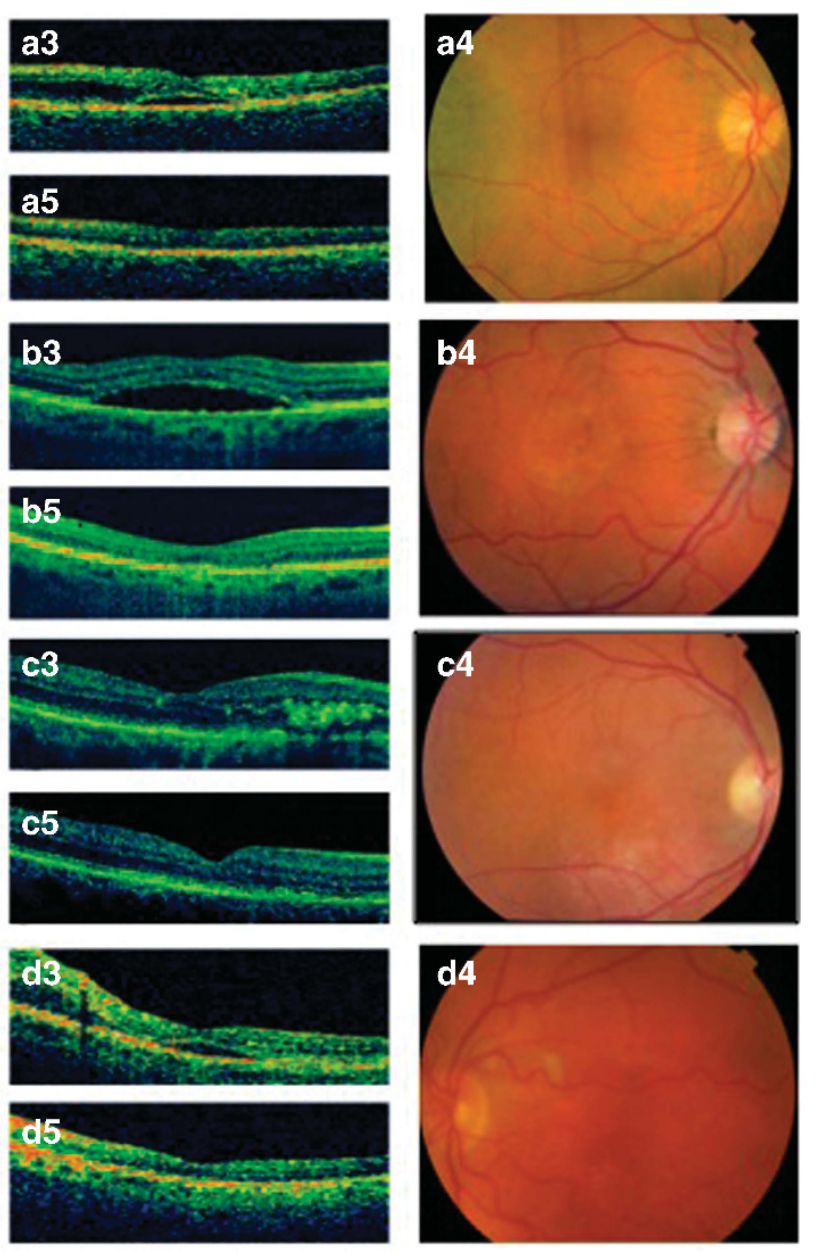

Figure 1 (a) Choroidal haemangioma: Pre-treatment colour photo (a.1), fluorescein angiogram (a.2), and OCT scan (a.3). Posttreatment colour photo (a.4) and OCT scan (a.5). (b) Central serous chorioretinopathy: Pre-treatment colour photo (b.1), fluorescein angiogram (b.2), and OCT scan (b.3). Post-treatment colour photo (b.4) and OCT scan (b.5). (c) Polypoidal choroidal vasculopathy: Pre-treatment colour photo (c.1), ICG angiogram (c.2), and OCT scan (c.3). Post-treatment colour photo (c.4) and OCT scan (c.5). (d) Peripapillary choroidal neovascularisation: Pre-treatment colour photo (d.1), fluorescein angiogram (d.2), and OCT scan (d.3). Post-treatment colour photo (d.4) and OCT scan (d.5). 
into two types based on clinical characteristics. ${ }^{41}$ Acute CSC tends to affect younger individuals with spontaneous resolution typically occurring within 3 months. Chronic or recurrent CSC tends to affect older individuals with a less favourable visual outcome. Progressive visual loss may develop due to foveal attenuation, cystoid macular degeneration, or damage to the foveal photoreceptor layer. Foveal photoreceptor atrophy can develop as early as 4 months after onset of symptoms.

CSC is initially managed conservatively as most cases will resolve spontaneously with visual recovery within a reasonable timeframe. In some cases, corticosteroid usage may be a contributory factor and stopping treatment should be considered (if possible). ${ }^{41}$ Laser photocoagulation was the traditional treatment for persistent CSC, but can potentially cause significant adverse effects (symptomatic scotomas, RPE atrophy, and secondary CNV). Micropulse diode laser with subthreshold therapy may be equally effective with reduced adverse effects. ${ }^{42}$ However, vPDT therapy is now generally considered to be the preferred treatment modality since it can directly target the principal abnormality of choroidal hyperpermeability. ${ }^{43,44}$

The proposed mechanism for vPDT therapy is closure of the abnormal leaking choroidal vessels with subsequent choroidal vascular remodelling. ${ }^{45}$ vPDT therapy is generally directed at the leakage site(s) identified on fluorescein angiography (Figure 1 b.1-b.5). It is possible that vPDT therapy might be better guided by indocyanine green (ICG) angiography, which typically demonstrates more widespread choroidal vascular abnormalities. ${ }^{46}$ Potential adverse effects of vPDT therapy include damage to the normal choriocapillaris and RPE leading to choroidal ischaemia, RPE atrophy and secondary CNV. These adverse effects assume greater significance when treating leakage site(s) close to the fovea.

CSC can be effectively treated using the standard vPDT protocol-verteporfin dosage $6 \mathrm{mg} / \mathrm{m}^{2}$ BSA and vPDT laser fluence $50 \mathrm{~J} / \mathrm{cm}^{2}\left(600 \mathrm{~mW} / \mathrm{cm}^{2}\right.$ for $\left.83 \mathrm{~s}\right) .{ }^{47}$ Alternative safety-enhanced vPDT protocols have been developed to provide effective treatment with reduced potential for adverse effects. Two principal approaches have been adopted as the photochemical response in the choroid is dose-response-dependent. Half-dose vPDT therapy uses a verteporfin dosage of $3 \mathrm{mg} / \mathrm{m}^{2}$ BSA. ${ }^{13,48-52}$ Reduced fluence vPDT therapy uses a vPDT laser fluence of $25 \mathrm{~J} / \mathrm{cm}^{2}\left(300 \mathrm{~mW} / \mathrm{cm}^{2}\right.$ for $\left.83 \mathrm{~s}\right) .{ }^{14,15,53,54}$ There is limited evidence to compare these different vPDT protocols for CSC in the absence of randomised controlled trials. ${ }^{44,55-58}$

The current approach to management of CSC is largely empirical. There is good evidence that vPDT therapy is a useful treatment option, but published studies are limited by selection bias, lack of randomisation, small sample size, and short follow-up. ${ }^{41,58}$ Selection of patients for vPDT therapy should be guided by factors such as duration, severity of visual symptoms and location of leakage site(s) relative to the fovea. The optimum vPDT protocol in respect of efficacy and safety remains to be determined. There is a need for robust clinical trials to investigate the role of vPDT therapy in the treatment of CSC, considering both the visual outcome of the treated episode and the recurrence rate in the longer term. It is noteworthy that the FDA granted orphan drug designation to verteporfin for the treatment of chronic or recurrent CSC in September 2012, which may help to drive future research.

\section{Polypoidal choroidal vasculopathy}

Polypoidal choroidal vasculopathy (PCV) is an exudative maculopathy characterised by an inner choroidal branching vascular network (BVN) with nodular polypoidal lesions. ${ }^{59}$ The aetiology is uncertain but arteriosclerosis of the choroidal vessels appears to be an important pathological feature. The typical clinical presentation is recurrent episodes of serous and/or haemorrhagic pigment epithelium detachment. The polyps may be visible as orange-red subretinal nodules. Diagnosis of PCV requires ICG angiography. It is defined as single or multiple focal nodular areas of hyperfluorescence arising from the choroidal circulation in the early phase (with or without visualisation of an associated BVN). ${ }^{60}$ There is a strong racial predilection for Asians and Africans compared with Caucasians. PCV is generally considered to be a distinct clinical entity (rather than a variant of neovascular AMD).

The best approach to treatment of PCV remains uncertain. Spontaneous resolution with visual recovery can occur in some cases. ${ }^{61}$ ICG-guided laser photocoagulation of active (leaking) polyps has been used in the past with variable success rates. ${ }^{62,63}$ Current methods of treatment involve the use of vPDT therapy and/or anti-VEGF therapy. ICG-guided vPDT treatment of active (leaking) polyps can resolve macular oedema through direct occlusion of polyps. ${ }^{64-66}$ Complete polyp regression is usually achieved with 1 or 2 treatments (Figure 1 c.1-c.5). The main adverse event is subretinal haemorrhage, which is usually limited and resolves without significant visual deterioration. ${ }^{64,67}$ Anti-VEGF therapy can also be beneficial in the treatment of PCV. It can reduce macular oedema, but has a variable effect on polyp regression. ${ }^{68,69}$ The EVEREST study demonstrated that vPDT therapy, either alone or in combination with ranibizumab, is better than ranibizumab monotherapy. ${ }^{70}$ 
Evidence-based guidelines for diagnosis and treatment of PCV have recently been developed. ${ }^{60}$ These were based on a systematic literature review (including the EVEREST study as the only published randomised controlled trial) and expert opinion. Recommended initial treatment is ICG-guided vPDT monotherapy or combination therapy with vPDT and ranibizumab (comprising three intravitreal injections of ranibizumab $0.5 \mathrm{mg}$ 1-month apart). If there is incomplete regression of polyps by ICG angiography, then further treatment should comprise vPDT monotherapy or combination therapy with vPDT and ranibizumab. If there is complete regression of polyps by ICG angiography but leakage on fluorescein angiography and other clinical or anatomical signs of disease activity, then further treatment should comprise ranibizumab.

Diagnosis (requiring ICG angiography) is probably the most important factor influencing successful treatment of PCV. A proportion of patients with clinical features suggestive of neovascular AMD will instead have PCV. The prevalence is estimated to be $5-10 \%$ among Caucasian patients and $20-50 \%$ among Asian patients. ${ }^{60,71}$ Further research is required to determine the optimum treatment for PCV. Current guidelines recommend the standard vPDT protocol-verteporfin dosage $6 \mathrm{mg} / \mathrm{m}^{2}$ BSA and vPDT laser fluence $50 \mathrm{~J} / \mathrm{cm}^{2}$ $\left(600 \mathrm{~mW} / \mathrm{cm}^{2}\right.$ for $\left.83 \mathrm{~s}\right){ }^{70}$ There is limited evidence for using alternative safety-enhanced vPDT protocols in PCV (such as half-dose or reduced fluence vPDT). Current guidelines recommend vPDT treatment of the entire PCV lesion (polyps plus BVN) rather than only the active (leaking) polyps. ${ }^{60}$ Further research is also required to define the role of anti-VEGF agents (to reduce leakage from polyps and the BVN) in combination therapy for PCV.

\section{Peripapillary choroidal neovascularisation}

Peripapillary CNV (PP-CNV) is defined as CNV located within one disc diameter of the optic nerve head. This condition is often idiopathic, but it can also occur secondary to inflammatory conditions (such as serpiginous choroidopathy or presumed ocular histoplasmosis syndrome) and optic nerve malformations (such as optic disc drusen or optic disc coloboma). PP-CNV becomes symptomatic owing to macular involvement from fluid exudation or haemorrhage. In some cases, the PP-CNV lesion can enlarge leading to direct foveal involvement.

Asymptomatic PP-CNV can be managed conservatively (particularly if located outside the macular arcade vessels) as spontaneous involution will often occur. Laser photocoagulation was the traditional treatment for symptomatic PP-CNV but carries a risk of thermal injury to the overlying neurosensory retina with consequent visual loss. The current treatment modalities comprise vPDT therapy or anti-VEGF therapy. ${ }^{72}$ vPDT therapy usually achieves PP-CNV regression following 1 or 2 treatments with only a small risk of an adverse effect (Figure 1 d.1-d.5). ${ }^{73,74}$ Anti-VEGF therapy is also effective but may require multiple injections to achieve PP-CNV regression. ${ }^{75,76}$

Most reports of vPDT therapy for PP-CNV have used the standard vPDT protocol-verteporfin dosage $6 \mathrm{mg} / \mathrm{m}^{2}$ BSA and vPDT laser fluence $50 \mathrm{~J} / \mathrm{cm}^{2}\left(600 \mathrm{~mW} / \mathrm{cm}^{2}\right.$ for $83 \mathrm{~s}$ ). Safety-enhanced vPDT protocols (such as half-dose or reduced fluence vPDT) may be equally effective since PP-CNV lesions tend to be less aggressive than subfoveal CNV lesions. When treating neovascular AMD, it has been advocated that the vPDT laser spot should not extend closer than $200 \mu \mathrm{m}$ to the border of the optic nerve head in order to avoid optic nerve damage. There is, however, evidence that the optic nerve head can be included in the treatment zone for PP-CNV lesions without causing optic nerve damage. ${ }^{74}$

\section{Conclusion}

vPDT therapy is a selective vaso-occlusive treatment that targets choroidal vascular abnormalities. It was initially developed to treat neovascular AMD with subfoveal CNV. The TAP and VIP studies (published 2001) demonstrated that vPDT was a moderately effective treatment for neovascular AMD with a good safety profile. vPDT therapy became the standard treatment for neovascular AMD until replaced by anti-VEGF therapy following the MARINA and ANCHOR studies (published 2006). Subsequently, vPDT therapy has evolved as an important treatment modality for a range of other chorioretinal conditions including choroidal haemangioma, central serous chorioretinopathy, polypoidal choroidal vasculopathy, and peripapillary choroidal neovascularisation. Published studies are, however, generally limited by selection bias, lack of randomisation, small sample size and short follow-up. Further research is required to optimise the vPDT protocols used to treat these chorioretinal conditions.

\section{Conflict of interest}

The author declares no conflict of interest.

\section{References}

1 Dougherty TJ, Gomer CJ, Henderson BW, Jori G, Kessel D, Korbelik $\mathrm{M}$ et al. Photodynamic therapy. J Natl Cancer Inst 1998; 90: 889-905.

2 Vrouenraets MB, Visser GW, Snow GB, van Dongen GA. Basic principles, applications in oncology and improved 
selectivity of photodynamic therapy. Anticancer Res 2003; 23: 505-522.

3 Brown SB, Brown EA, Walker I. The present and future role of photodynamic therapy in cancer treatment. Lancet Oncol 2004; 5: 497-508.

4 Regillo CD. Update on photodynamic therapy. Curr Opin Ophthalmol 2000; 11: 166-170.

5 Miller JW, Schmidt-Erfurth U, Sickenberg M, Pournaras CJ, Laqua $\mathrm{H}$, Barbazetto I et al. Photodynamic therapy with verteporfin for choroidal neovascularization caused by age-related macular degeneration: results of a single treatment in a phase 1 and 2 study. Arch Ophthalmol 1999; 117: 1161-1173.

6 Schmidt-Erfurth U, Miller JW, Sickenberg M, Laqua H, Barbazetto I, Gragoudas ES et al. Photodynamic therapy with verteporfin for choroidal neovascularization caused by age-related macular degeneration: results of retreatments in a phase 1 and 2 study. Arch Ophthalmol 1999; 117: 1177-1187.

7 Miller JW, Walsh AW, Kramer M, Hasan T, Michaud N, Flotte TJ et al. Photodynamic therapy of experimental choroidal neovascularization using lipoprotein-delivered benzoporphyrin. Arch Ophthalmol 1995; 113: 810-818.

8 Schmidt-Erfurth U, Hasan T. Mechanisms of action of photodynamic therapy with verteporfin for the treatment of age-related macular degeneration. Surv Ophthalmol 2000; 45: 195-214.

9 Kramer M, Miller JW, Michaud N, Moulton RS, Hasan T, Flotte TJ et al. Liposomal benzoporphyrin derivative verteporfin photodynamic therapy. Selective treatment of choroidal neovascularization in monkeys. Ophthalmology 1996; 103: 427-438.

10 Treatment of Age-Related Macular Degeneration with Photodynamic Therapy (TAP) Study Group. Photodynamic therapy of subfoveal choroidal neovascularization in agerelated macular degeneration with verteporfin: two-year results of 2 randomized clinical trials. TAP report 2. Arch Ophthalmol 2001; 119: 198-207.

11 Verteporfin In Photodynamic Therapy Study Group. Verteporfin therapy of subfoveal choroidal neovascularization in age-related macular degeneration: two-year results of a randomized clinical trial including lesions with occult with no classic choroidal neovascularization. Verteporfin in photodynamic therapy report 2. Am J Ophthalmol 2001; 131: 541-560.

12 Visudyne in Minimally Classic Choroidal Neovascularization Study Group. Verteporfin therapy of subfoveal minimally classic choroidal neovascularization in age-related macular degeneration: 2-year results of a randomized clinical trial. Arch Ophthalmol 2005; 123: 448-457.

13 Chan WM, Lai TY, Lai RY, Tang EW, Liu DT, Lam DS. Safety enhanced photodynamic therapy for chronic central serous chorioretinopathy: one-year results of a prospective study. Retina 2008; 28: 85-93.

14 Shin JY, Woo SJ, Yu HG, Park KH. Comparison of efficacy and safety between half-fluence and full-fluence photodynamic therapy for chronic central serous chorioretinopathy. Retina 2011; 31: 119-126.

15 Reibaldi M, Boscia F, Avitabile T, Uva MG, Russo A, Zagari $\mathrm{M}$ et al. Functional retinal changes measured by microperimetry in standard-fluence vs low-fluence photodynamic therapy in chronic central serous chorioretinopathy. Am J Ophthalmol 2011; 151: 953-960.
16 Rosenfeld PJ, Brown DM, Heier JS, Boyer DS, Kaiser PK, Chung CY et al. MARINA Study Group. Ranibizumab for neovascular age-related macular degeneration. $N$ Engl J Med 2006; 355: 1419-1431.

17 Brown DM, Kaiser PK, Michels M, Soubrane G, Heier JS, Kim RY et al. ANCHOR Study Group. Ranibizumab versus verteporfin for neovascular age-related macular degeneration. N Engl J Med 2006; 355: 1432-1444.

18 Brown DM, Michels M, Kaiser PK, Heier JS, Sy JP, Ianchulev T. ANCHOR Study Group. Ranibizumab versus verteporfin photodynamic therapy for neovascular agerelated macular degeneration: Two-year results of the ANCHOR study. Ophthalmology 2009; 116: 57-65.

19 Larsen M, Schmidt-Erfurth U, Lanzetta P, Wolf S, Simader C, Tokaji E et al. MONT BLANC Study Group. Verteporfin plus ranibizumab for choroidal neovascularization in age-related macular degeneration: twelve-month MONT BLANC study results. Ophthalmology 2012; 119: 992-1000.

20 Kaiser PK, Boyer DS, Cruess AF, Slakter JS, Pilz S, Weisberger A. DENALI Study Group. Verteporfin plus ranibizumab for choroidal neovascularization in age-related macular degeneration: twelve-month results of the DENALI study. Ophthalmology 2012; 119: 1001-1010.

21 Tozer K, Roller AB, Chong LP, Sadda S, Folk JC, Mahajan VB et al. Combination therapy for neovascular age-related macular degeneration refractory to anti-vascular endothelial growth factor agents. Ophthalmology 2013; 120: 2029-2034.

22 Sickenberg M, Schmidt-Erfurth U, Miller JW, Pournaras CJ, Zografos L, Piguet B et al. A preliminary study of photodynamic therapy using verteporfin for choroidal neovascularization in pathologic myopia, ocular histoplasmosis syndrome, angioid streaks, and idiopathic causes. Arch Ophthalmol 2000; 118: 327-336.

23 Verteporfin in Photodynamic Therapy Study Group. Photodynamic therapy of subfoveal choroidal neovascularization in pathologic myopia with verteporfin. 1 -year results of a randomized clinical trial. VIP Report No 1. Ophthalmology 2001; 108: 841-852.

24 Blinder KJ, Blumenkranz MS, Bressler NM, Bressler SB, Donato G, Lewis $\mathrm{H}$ et al. Verteporfin therapy of subfoveal choroidal neovascularization in pathologic myopia: 2-year results of a randomized clinical trial. VIP Report No 3. Ophthalmology 2003; 110: 667-673.

25 Ruiz-Moreno JM, Amat P, Montero JA, Lugo F. Photodynamic therapy to treat choroidal neovascularisation in highly myopic patients: 4 years' outcome. $\mathrm{Br} J$ Ophthalmol 2008; 92: 792-794.

26 Hayashi K, Ohno-Matsui K, Teramukai S, Shimada N, Moriyama M, Hayashi W et al. Comparison of visual outcome and regression pattern of myopic choroidal neovascularization after intravitreal bevacizumab or after photodynamic therapy. Am J Ophthalmol 2009; 148: 396-408.

27 Baba T, Kubota-Taniai M, Kitahashi M, Okada K, Mitamura Y, Yamamoto S. Two-year comparison of photodynamic therapy and intravitreal bevacizumab for treatment of myopic choroidal neovascularisation. Br J Ophthalmol 2010; 94: 864-870.

28 Wolf S, Balciuniene VJ, Laganovska G, Menchini U, OhnoMatsui K, Sharma T et al. RADIANCE Study Group. RADIANCE: a randomized controlled study of ranibizumab in patients with choroidal neovascularization secondary to pathologic myopia. Ophthalmology 2014; 121: 682-692.

29 Wong TY, Ohno-Matsui K, Leveziel N, Holz FG, Lai TY, $\mathrm{Yu}$ HG et al. Myopic choroidal neovascularisation: current 
concepts and update on clinical management. Br J Ophthalmol 2015; 99: 289-296.

30 Verteporfin Roundtable 2000 and 2001 Participants. Guidelines for using verteporfin (visudyne) in photodynamic therapy to treat choroidal neovascularization due to age-related macular degeneration and other causes. Retina 2002; 22: 6-18.

31 Heier JS, Brown D, Ciulla T, Abraham P, Bankert JM, Chong $S$ et al. Ranibizumab for choroidal neovascularization secondary to causes other than age-related macular degeneration: a phase I clinical trial. Ophthalmology 2011; 118: 111-118.

32 Kang HM, Koh HJ. Intravitreal anti-vascular endothelial growth factor therapy versus photodynamic therapy for idiopathic choroidal neovascularization. Am J Ophthalmol 2013; 155: 713-719.

33 Schmidt-Erfurth UM, Michels S, Kusserow C, Jurklies B, Augustin AJ. Photodynamic therapy for symptomatic choroidal hemangioma: visual and anatomic results. Ophthalmology 2002; 109: 2284-2294.

34 Jurklies B, Anastassiou G, Ortmans S, Schüler A, Schilling H, Schmidt-Erfurth $\mathrm{U}$ et al. Photodynamic therapy using verteporfin in circumscribed choroidal haemangioma. Br J Ophthalmol 2003; 87: 84-89.

35 Porrini G, Giovannini A, Amato G, Ioni A, Pantanetti M. Photodynamic therapy of circumscribed choroidal hemangioma. Ophthalmology 2003; 110: 674-680.

36 Singh AD, Kaiser PK, Sears JE, Gupta M, Rundle PA, Rennie IG. Photodynamic therapy of circumscribed choroidal haemangioma. Br J Ophthalmol 2004; 88: 1414-1418.

37 Boixadera A, García-Arumí J, Martínez-Castillo V, Encinas JL, Elizalde J, Blanco-Mateos G et al. Prospective clinical trial evaluating the efficacy of photodynamic therapy for symptomatic circumscribed choroidal hemangioma. Ophthalmology 2009; 116: 100-105.

38 Blasi MA, Tiberti AC, Scupola A, Balestrazzi A, Colangelo E, Valente $\mathrm{P}$ et al. Photodynamic therapy with verteporfin for symptomatic circumscribed choroidal hemangioma: fiveyear outcomes. Ophthalmology 2010; 117: 1630-1637.

39 Frau E, Rumen F, Noel G, Delacroix S, Habrand JL, Offret H. Low-dose proton beam therapy for circumscribed choroidal hemangiomas. Arch Ophthalmol 2004; 122: 1471-1475.

40 Tsipursky MS, Golchet PR, Jampol LM. Photodynamic therapy of choroidal hemangioma in sturge-weber syndrome, with a review of treatments for diffuse and circumscribed choroidal hemangiomas. Surv Ophthalmol 2011; 56: 68-85.

41 Ross A, Ross AH, Mohamed Q. Review and update of central serous chorioretinopathy. Curr Opin Ophthalmol 2011; 22: 166-173.

42 Lanzetta P, Furlan F, Morgante L, Veritti D, Bandello F. Nonvisible subthreshold micropulse diode laser $(810 \mathrm{~nm})$ treatment of central serous chorioretinopathy. A pilot study. Eur J Ophthalmol 2008; 18: 934-940.

43 Maruko I, Iida T, Sugano Y, Ojima A, Ogasawara M, Spaide RF. Subfoveal choroidal thickness after treatment of central serous chorioretinopathy. Ophthalmology 2010; 117: 1792-1799.

44 Ma J, Meng N, Xu X, Zhou F, Qu Y. System review and meta-analysis on photodynamic therapy in central serous chorioretinopathy. Acta Ophthalmol 2014; 92: e594-e601.

45 Chan WM, Lam DS, Lai TY, Tam BS, Liu DT, Chan CK. Choroidal vascular remodelling in central serous chorioretinopathy after indocyanine green guided photodynamic therapy with verteporfin: a novel treatment at the primary disease level. Br J Ophthalmol 2003; 87: 1453-1458.

46 Yannuzzi LA, Slakter JS, Gross NE, Spaide RF, Costa D, Huang SJ et al. Indocyanine green angiography-guided photodynamic therapy for treatment of chronic central serous chorioretinopathy: a pilot study. Retina 2003; 23: 288-298.

47 Silva RM, Ruiz-Moreno JM, Gomez-Ulla F, Montero JA, Gregório T, Cachulo ML et al. Photodynamic therapy for chronic central serous chorioretinopathy: a 4-year follow-up study. Retina 2013; 33: 309-315.

48 Chan WM, Lai TY, Lai RY, Liu DT, Lam DS. Half-dose verteporfin photodynamic therapy for acute central serous chorioretinopathy: one-year results of a randomized controlled trial. Ophthalmology 2008; 115: 1756-1765.

49 Fujita K, Yuzawa M, Mori R. Retinal sensitivity after photodynamic therapy with half-dose verteporfin for chronic central serous chorioretinopathy: short-term results. Retina 2011; 31: 772-778.

50 Karakus SH, Basarir B, Pinarci EY, Kirandi EU, Demirok A. Long-term results of half-dose photodynamic therapy for chronic central serous chorioretinopathy with contrast sensitivity changes. Eye 2013; 27: 612-620.

51 Tseng CC, Chen SN. Long-term efficacy of half-dose photodynamic therapy on chronic central serous chorioretinopathy. Br J Ophthalmol 2015; 99: 1070-1077.

52 Fujita K, Imamura Y, Shinoda K, Matsumoto CS, Mizutani Y, Hashizume $\mathrm{K}$ et al. One-year outcomes with half-dose verteporfin photodynamic therapy for chronic central serous chorioretinopathy. Ophthalmology 2015; 122: 555-561.

53 Reibaldi M, Cardascia N, Longo A, Furino C, Avitabile T, Faro $\mathrm{S}$ et al. Standard-fluence versus low-fluence photodynamic therapy in chronic central serous chorioretinopathy: a nonrandomized clinical trial. Am J Ophthalmol 2010; 149: 307-315.

54 Smretschnig E, Ansari-Shahrezaei S, Hagen S, Glittenberg C, Krebs I, Binder S. Half-fluence photodynamic therapy in chronic central serous chorioretinopathy. Retina 2013; 33: 316-323.

55 Nicolo M, Eandi CM, Alovisi C, Grignolo FM, Traverso CE, Musetti D et al. Half-fluence versus half-dose photodynamic therapy in chronic central serous chorioretinopathy. Am J Ophthalmol 2014; 157: 1033-1037.

56 Alkin Z, Perente I, Ozkaya A, Alp D, Agca A, Aygit ED et al. Comparison of efficacy between low-fluence and half-dose verteporfin photodynamic therapy for chronic central serous chorioretinopathy. Clin Ophthalmol 2014; 8: 685-690.

57 Lim JI, Glassman AR, Aiello LP, Chakravarthy U, Flaxel CJ, Spaide RF. Macula Society CSC Collaborative Study Group. Collaborative retrospective macula society study of photodynamic therapy for chronic central serous chorioretinopathy. Ophthalmology 2014; 121: 1073-1078.

58 Erikitola OC, Crosby-Nwaobi R, Lotery AJ, Sivaprasad S. Photodynamic therapy for central serous chorioretinopathy. Eye 2014; 28: 944-957.

59 Gomi F, Tano Y. Polypoidal choroidal vasculopathy and treatments. Curr Opin Ophthalmol 2008; 19: 208-212.

60 Koh AH, Chen LJ, Chen SJ, Chen Y, Giridhar A, Iida T et al. Expert PCV Panel. Polypoidal choroidal vasculopathy: evidence-based guidelines for clinical diagnosis and treatment. Retina 2013; 33: 686-716. 
61 Uyama M, Wada M, Nagai Y, Matsubara T, Matsunaga H, Fukushima I et al. Polypoidal choroidal vasculopathy: natural history. Am J Ophthalmol 2002; 133: 639-648.

62 Yuzawa M, Mori R, Haruyama M. A study of laser photocoagulation for polypoidal choroidal vasculopathy. Jpn J Ophthalmol 2003; 47: 379-384.

63 Lee MW, Yeo I, Wong D, Ang CL. Argon laser photocoagulation for the treatment of polypoidal choroidal vasculopathy. Eye 2009; 23: 145-148.

64 Chan WM, Lam DS, Lai TY, Liu DT, Li KK, Yao Y et al. Photodynamic therapy with verteporfin for symptomatic polypoidal choroidal vasculopathy: one-year results of a prospective case series. Ophthalmology 2004; 111: 1576-1584.

65 Otani A, Sasahara M, Yodoi Y, Aikawa H, Tamura H, Tsujikawa A et al. Indocyanine green angiography: guided photodynamic therapy for polypoidal choroidal vasculopathy. Am J Ophthalmol 2007; 144: 7-14.

66 Eandi CM, Ober MD, Freund KB, Slakter JS, Yannuzzi LA. Selective photodynamic therapy for neovascular age-related macular degeneration with polypoidal choroidal neovascularization. Retina 2007; 27: 825-831.

67 Hirami Y, Tsujikawa A, Otani A, Yodoi Y, Aikawa H, Mandai $\mathrm{M}$ et al. Hemorrhagic complications after photodynamic therapy for polypoidal choroidal vasculopathy. Retina 2007; 27: 335-341.

68 Kokame GT, Yeung L, Lai JC. Continuous anti-VEGF treatment with ranibizumab for polypoidal choroidal vasculopathy: 6-month results. Br J Ophthalmol 2010; 94: 297-301.
69 Gomi F, Sawa M, Wakabayashi T, Sasamoto Y, Suzuki M, Tsujikawa M. Efficacy of intravitreal bevacizumab combined with photodynamic therapy for polypoidal choroidal vasculopathy. Am J Ophthalmol 2010; 150: $48-54$.

70 Koh A, Lee WK, Chen LJ, Chen SJ, Hashad Y, Kim H et al. EVEREST study: efficacy and safety of verteporfin photodynamic therapy in combination with ranibizumab or alone versus ranibizumab monotherapy in patients with symptomatic macular polypoidal choroidal vasculopathy. Retina 2012; 32: 1453-1464.

71 Davis SJ, Lauer AK, Flaxel CJ. Polypoidal choroidal vasculopathy in white patients. Retina 2014; 34: 2185-2191.

72 Jutley G, Jutley G, Tah V, Lindfield D, Menon G. Treating peripapillary choroidal neovascular membranes: a review of the evidence. Eye 2011; 25: 675-681.

73 Rosenblatt BJ, Shah GK, Blinder K. Photodynamic therapy with verteporfin for peripapillary choroidal neovascularization. Retina 2005; 25: 33-37.

74 Bernstein PS, Horn RS. Verteporfin photodynamic therapy involving the optic nerve for peripapillary choroidal neovascularization. Retina 2008; 28: 81-84.

75 Figueroa MS, Noval S, Contreras I. Treatment of peripapillary choroidal neovascular membranes with intravitreal bevacizumab. $\mathrm{Br}$ J Ophthalmol 2008; 92: 1244-1247.

76 Hoeh AE, Schaal KB, Ach T, Dithmar S. Treatment of peripapillary choroidal neovascularization with intravitreal bevacizumab. Eur J Ophthalmol 2009; 19: 163-165. 